\title{
Biointensive Management of Okra Fruit Borers using Braconid Parasitoids (Braconidae: Hymenoptera)
}

\author{
G. Thanavendan and S. Jeyarani \\ Department of Agricultural Entomology \\ Tamil Nadu Agricultural University \\ Coimbatore, Tamil Nadu \\ India
}

\begin{abstract}
Okra (Abelmoschus esculentus (L.) Moench) is ravaged by many insect pests from germination to harvest. Sucking pests in the early stage and the fruit borers, Earias vittella Fabricius and Helicoverpa armigera (Hübner) in the later stage causes extensive damage to fruits causing 69 per cent yield loss. In recent years, increased interest has been shown towards biological control following unsatisfactory results with the application of conventional insecticides. Horticultural crops, chiefly fruit crops, provide a stable environment offering good opportunity for biological control. Hence, investigations were carried out during 2007-2008 to assess the potential of braconid parasitoids against okra fruit borers. The results revealed that the combination of Trichogramma chilonis +

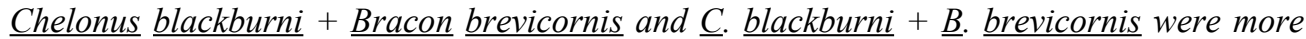
effective to lower larval population of $\underline{E}$. vittella and $\underline{H}$. armigera which was next to Endosulfan 35 EC after three releases/spray. Similar trend was also observed for per cent fruit damage on both number and weight basis. Combination of $\underline{T \text {. chilonis }}+\underline{C}$. blackburni $+\underline{B} . \underline{\text { brevicornis }}$ and $\underline{C} . \underline{\text { blackburni }}+\underline{B}$. brevicornis recorded higher yield which was on par with endosulfan $35 \mathrm{EC}$. The results of the trials widened the scope of utilizing the braconid parasitoids in the biosuppression of fruit borers on okra.
\end{abstract}

\section{INTRODUCTION}

Okra (Abelmoschus esculentus (L.) Moench) which belongs to Family Malvaceae is one of the important vegetable crops grown throughout the tropical and warm temperate regions of the world. In India, it is grown in both Kharif and summer season. Globally, okra is cultivated in an area of 0.78 million ha producing 4.99 million MT with an average yield of $6.39 \mathrm{t} \mathrm{ha}^{-1}$ (Gopalakrishnan, 2007). In India, it is cultivated in an area of 0.31 million ha producing 3.65 million MT with an average yield of $9.59 \mathrm{t} \mathrm{ha}^{-1}$ (FAO, 2007). In Tamil Nadu, it occupies an area of 4400 ha with an annual tender fruit production and productivity of 36,000 MT and $7.5 \mathrm{t} \mathrm{ha}^{-1}$, respectively (Gopalakrishnan, 2007). Okra is ravaged by many insect pests right from germination to harvest (Jagtab et al., 2007). Fruit borers, Earias vittella Fabricius and Earias insulana Boisdual in the later stages of crop growth causes extensive damage to fruits resulting in 69\% yield loss (Atwal and Singh, 1990). Helicoverpa armigera (Hübner), which was considered as one of the minor pests, has recently emerged as a major limiting factor in okra production. Its infestation affects the quality and quantity of fruit production, which ultimately reduce the market price. Both E. vittella and H. armigera causes up to $50 \%$ loss in okra in different parts of the country (Mani et al., 2005).

A wide range of recommended insecticides are being used by the farmers for controlling the pests (Priya and Misra, 2007). The chemicals are highly effective, rapid in action, adaptable 
to most situations and relatively economical. Despite these advantages, the use of chemical pesticides had been ecologically unsafe and harmful to natural enemies. The use of persistent insecticides on vegetables and fruits is a concern due to practical limitations on following pre-harvest interval. The increasing concern for environmental safety and global demand for pesticide free food necessitated the search for eco friendly methods of pest management. The alternative to tackle these problems is to develop a biocontrol strategy involving potential natural enemies which can be successfully incorporated into a sound Integrated Pest Management (IPM) programme. Horticultural crops, chiefly the fruits, provide stable environment offering good opportunity for biological control in the pest management programme. Among the various groups of biocontrol agents, braconids are well known parasitoids for the management of different lepidopteran larvae, including okra fruit borer complex. King et al., (1985) reported that field release of the braconid parasitoid, Chelonus blackburni Cameron at 50,000 adults ha $^{-1}$ gave promising control of $E$. vittella with least fruit infestation of $11.64 \%$. Mani et al., (2005) reported the natural incidence of $B$. hebetor, $B$. greeni and Trichogramma spp., on Earias spp. in okra fields. However, information on the efficacy of braconids on okra fruit borers is very scarce. Keeping these in view, the present investigations were made to evaluate the efficacy of braconid parasitoids in comparison with already available recommended practices against the okra fruit borers under field conditions.

\section{MATERIALS AND METHODS}

\section{Culture of parasitoids}

The larval parasitoid, Bracon brevicornis Wesmael (Braconidae: Hymenoptera) was obtained from the Central Plantation Crops Research Institute (CPCRI), Kayankulam, Kerala District and were maintained in the Biocontrol Laboratory, Department of Agricultural Entomology, Tamil Nadu Agricultural University (TNAU), Coimbatore following the procedures adopted by Jhansi (1984). The egg larval parasitoid C. blackburni (Braconidae: Hymenoptera) and the egg parasitoid, Trichogramma chilonis Ishii (Trichogrammatidae: Hymenoptera) maintained in the Biocontrol Laboratory, TNAU were utilized for the field experiments.

\section{Parasitic potential of B. brevicornis and C. blackburni against fruit borers}

An experiment was designed to examine the effects of different parasitoid densities and host age on per cent parasitization. The second, third and fourth instar larvae of E. vittella were introduced separately at different ratios of parasitoids and host viz., 1:10, 2:10, 3:10, 4:10 and 5:10. In all the cases, mated females were used and the experiment was carried out in a plastic jar of $18 \times 12 \mathrm{~cm}$ size, by sandwich method (Jhansi, 1984) with four replications. After $24 \mathrm{~h}$, the larvae were observed and the per cent parasitization was calculated. A similar study was carried out for the second, third, fourth and fifth instar larvae of H. armigera. In order to examine the parasitic potential of C. blackburni, another experiment was carried out with different ratios of its adults and eggs of E. vittella and H. armigera by adopting the procedure of Swamiappan and Balasubramanian (1979). The parasitoid and host eggs were maintained at a ratio of $1: 100,2: 100,3: 100,4: 100$ and 5:100 with four replications. The observation on per cent parasitization was recorded after the egg incubation period.

\section{Field evaluation of braconid parasitoids}

To evaluate the efficacy of the braconid parasitoids viz., B. brevicornis and C. blackburni against $E$. vittella and $H$. armigera on okra, two field experiments were conducted at 
Vellimalaipatinam and Kozhimadai villages of Thondamuthur and Madhukkarai block, Coimbatore district, Tamil Nadu. The varieties, Mahyco-10 and U.S Agriseeds were used for the trials. The release rates of parasitoids were decided based on the parasitic potential studies.

The treatments included in the experiment were as follows.

1. B. brevicornis alone @ 2000 adults ha ${ }^{-1}$

2. T. chilonis@1,00,000 adults ha ${ }^{-1}$

3. C.blackburni@2000 adults ha ${ }^{-1}$

4. Neem Seed Kernel Extract (NSKE) 5\%

5. T. chilonis + B. brevicornis @ half the dose each

6. C. blackburni + B. brevicornis@ half the dose each

7. NSKE + B. brevicornis @ half the dose each

8. T. chilonis + C. blackburni + B. brevicornis @ half the dose each

9. Endosulfan 35EC @ $1000 \mathrm{ml} \mathrm{ha}^{-1}$

10. Untreated check.

The treatments were replicated thrice with a plot size of $4 \times 5 \mathrm{~m}$. Treatments were imposed at 15 day intervals three times. The sachet made up of tissue paper containing ready to emerge cocoons of B. brevicornis and adults of C. blackburni were tied individually in the middle of the treatment plots. The mouth of the sachet was tied after inserting a small piece of straw to facilitate the exit of adults. In between each treatment and each replication, a buffer plot sprayed with chemical at weekly intervals was maintained to restrict the movement of the parasitoids from the released plots. The insecticide, endosulfan recommended in the package of practice of Tamil Nadu Agricultural University, Coimbatore, Tamil Nadu, India was used as a standard for comparison. Starting at forty five days after sowing, observations on the population of $E$. vittella and $H$. armigera were recorded in ten randomly selected plants from each plot before and after 4,8 , and 12 days of treatment and the pooled mean was worked out after three rounds of release/spray.

\section{Assessment of fruit borer damage and yield}

Borer damage in okra fruits was recorded from ten randomly selected plants for each treatment and replications. The pre treatment counts were made on the total number of fruits and affected fruits. Subsequently, counts were made during each picking at an interval of 4 days. The per cent damage due to fruit borers was worked out on both number and weight basis and pooled mean was worked out after three rounds of release/spray. During each picking, the borers affected okra fruits from ten plants in each treatment were brought to the laboratory and observations on the mortality of the larvae due to parasitization or chemical were assessed. The parasitoids thus emerged were released again in the respective treatment plots.

The yield data was also worked out and expressed as $\mathrm{kg} \mathrm{ha}^{-1}$. Yield was analyzed statistically after pooling the data from every picking and the cost: benefit ratio was worked out as per the following formula (Akila and Babu, 1994).

Cost: Benefit ratio $=\frac{\text { Value of produce }}{\text { Cost of cultivation }+ \text { Cost of plant protection }}$ 
The larval counts in the field experiments were transformed to values as per method developed by Poisson for statistical analysis (Snedecor and Cochran, 1967). The data on per cent fruit borer damage was analyzed statistically after arc sin transformation.

\section{RESULTS AND DISCUSSION}

\section{Parasitic potential of $\boldsymbol{B}$. brevicornis and $\boldsymbol{C}$. blackburni against fruit borers}

The parasitic potential studies showed that the parasitoid host ratio of 5:10 was the best for both $E$. vittella and $H$. armigera with the highest parasitization against all instars tested (Table 1). Also, B. brevicornis was more effective against $E$. vittella followed by $H$. armigera. Sheeba and Narendran (2007) reported that preferences of the parasitoid vary with host insects. According to them, B. brevicornis was more effective against $O$. arenosella with $90 \%$ parasitization.

Table 1. Parasitic potential of B. brevicornis against the larvae of $\boldsymbol{E}$. vittella and $\boldsymbol{H}$. armigera

\begin{tabular}{|c|c|c|c|c|c|c|}
\hline \multirow{3}{*}{$\begin{array}{l}\text { Parasitoid: } \\
\text { host ratio* }\end{array}$} & \multicolumn{6}{|c|}{ Per cent Parasitization $(\%) *$} \\
\hline & \multicolumn{3}{|c|}{ E. vittella } & \multicolumn{3}{|c|}{ H. armigera } \\
\hline & II instar & III instar & IV instar & II instar & III instar & IV instar $V$ instar \\
\hline \multirow{2}{*}{$1: 10$} & 45.00 & 55.00 & 57.50 & 40.50 & 50.00 & 52.00 \\
\hline & $(42.13)^{\mathrm{e}}$ & $(48.16)^{d}$ & $(49.31)^{\mathrm{d}}$ & $(39.52)^{d}$ & $(45.00)^{\mathrm{d}}$ & $(46.15)^{\mathrm{c}}(30.52)^{\mathrm{e}}$ \\
\hline \multirow{2}{*}{ 2:10 } & 55.00 & 67.50 & 62.00 & 70.00 & 68.73 & $\begin{array}{ll}75.00 & 45.11\end{array}$ \\
\hline & $(47.87)^{\mathrm{d}}$ & $(55.24)^{\mathrm{c}}$ & $(51.94)^{\mathrm{c}}$ & $(56.78)^{\mathrm{c}}$ & $(55.98)^{\mathrm{c}}$ & $(60.00)^{b}(42.19)^{d}$ \\
\hline \multirow{2}{*}{$3: 10$} & 75.00 & 85.00 & 95.50 & 83.15 & 90.00 & 100.00 \\
\hline & $(60.00)^{\mathrm{c}}$ & $(67.21)^{\mathrm{b}}$ & $(77.75)^{b}$ & $(65.76)^{\mathrm{b}}$ & $(71.56)^{b}$ & $(90.00)^{\mathrm{a}}(52.53)^{\mathrm{c}}$ \\
\hline \multirow{2}{*}{$4: 10$} & 90.00 & 100.00 & 100.00 & 100.00 & 100.00 & 100.00 \\
\hline & $(71.56)^{\mathrm{b}}$ & $(90.00)^{\mathrm{a}}$ & $(90.00)^{\mathrm{a}}$ & $(90.00)^{\mathrm{a}}$ & $(90.00)^{\mathrm{a}}$ & $(90.00)^{\mathrm{a}}(59.67)^{\mathrm{b}}$ \\
\hline \multirow{2}{*}{$5: 10$} & 100.00 & 100.00 & 100.00 & 100.00 & 100.00 & 100.00 \\
\hline & $(90.00)^{\mathrm{a}}$ & $(90.00)^{\mathrm{a}}$ & $(90.00)^{\mathrm{a}}$ & $(90.00)^{\mathrm{a}}$ & $(90.00)^{\mathrm{a}}$ & $(90.00)^{\mathrm{a}}(68.86)^{\mathrm{a}}$ \\
\hline
\end{tabular}

* Mean of four replications in each treatment

** Figures in parentheses are arc sin values

In a column, means followed by same letter(s) are not significantly different by DMRT $(\mathrm{P}<0.05)$

In the present investigation, it was also observed that the fourth instar larvae was preferred by the parasitoids than early instars. The host acceptance and per cent parasitization may vary according to the age of the host. Hopper (1986) reported that M. croceipes preferred third and fourth instar larvae of Heliothis virescens (F.). However, early instars were also parasitized. It is in confirmation with earlier findings of Reichmuth et al., (1997). According to them, very young and small lepidopteran hosts can also be killed when stung by the parasitoids. Results of the studies on parasitic potential of C. blackburni showed that the highest parasitization of 64.58 and $70.54 \%$ was recorded against $E$. vittella and $H$. armigera, respectively at a parasitoid host ratio of 5:100 (Table 2).

Table 2. Parasitic potential of $C$. blackburni against $E$. vittella and $H$. armigera eggs

\begin{tabular}{|c|c|c|}
\hline \multirow{2}{*}{ Parasitoid: Host ratio* } & \multicolumn{2}{|c|}{ Parasitization $(\%)^{* *}$} \\
\hline & E. vittella & H. armigera \\
\hline $1: 100$ & $25.60(30.39)^{\mathrm{e}}$ & $28.40(32.20)^{\mathrm{e}}$ \\
\hline $2: 100$ & $38.31(38.22)^{\mathrm{d}}$ & $43.48(41.24)^{\mathrm{d}}$ \\
\hline $3: 100$ & $52.81(46.61)^{c}$ & $58.07(49.65)^{\mathrm{c}}$ \\
\hline $4: 100$ & $60.00(50.76)^{b}$ & $64.85(53.65)^{\mathrm{b}}$ \\
\hline $5: 100$ & $64.58(53.49)^{\mathrm{a}}$ & $70.54(57.13)^{\mathrm{a}}$ \\
\hline
\end{tabular}

* Mean of four replications in each treatment 
** Figures in parentheses are arc sin values

In a column, means followed by same letter(s) are not significantly different by DMRT $(\mathrm{P}<0.05)$

In agreement with the results of the present study Swamiappan and Balasubramanian (1979) reported 59.60\% parasitization against E. vittella and Jeyarani et al., (2008) reported 87.11\% parasitization against $H$. armigera by $C$. blackburni.

\section{Field evaluation of braconid parasitoids}

Observations on the larval population showed that the larvae of E. vittella preferred to feed on freshly formed and tender fruits, while, $H$. armigera did not show any preference for the stage of fruits. However, the larvae of both species were rarely observed together in a single fruit. Results of both the trials showed that combination of two braconid parasitoids viz., $C$. blackburni + B. brevicornis were more effective and were on par with combination of $T$. chilonis + C. blackburni + B. brevicornis next to endosulfan in the order of efficacy in reducing the larval population and fruit damage. In the first trial, among the treatments, combination of $T$. chilonis $+C$. blackburni + B. brevicornis and C. blackburni $+B$. brevicornis recorded the lowest pooled mean larval population of $E$. vittella and were on par with each other, recording 40.65 and $37.34 \%$ reduction over control which was next to endosulfan $35 \mathrm{EC}$ in the order of efficacy after three releases/spray. Similar trend was also observed against $H$. armigera (Table 3).

Table 3. Efficacy of braconid parasitoids against larval population of okra fruit borers: trial I (Vellimalaipatinam, Coimbatore 2007 - 08)

\begin{tabular}{|c|c|c|c|c|c|c|}
\hline \multirow[t]{2}{*}{ Treatment* } & \multicolumn{4}{|c|}{$\begin{array}{l}\text { No. of larvae } / 10 \text { plants } \\
\quad(\text { pooled mean)** }\end{array}$} & \multicolumn{2}{|c|}{$\begin{array}{c}\% \text { reduction over PTC } \\
\text { (due to parasitization / } \\
\text { chemicals) }\end{array}$} \\
\hline & PTC & E. vittella & PTC & H. armigera & E. vittella & H. armigera \\
\hline B. brevicornis & 10.85 & $\begin{array}{c}8.02 \\
(2.92)^{\mathrm{c}}\end{array}$ & 12.00 & $\begin{array}{c}8.26 \\
(2.96)^{\mathrm{b}}\end{array}$ & 26.08 & 31.17 \\
\hline T. chilonis & 11.15 & $\begin{array}{c}8.31 \\
(2.97)^{\mathrm{c}}\end{array}$ & 12.41 & $\begin{array}{c}8.61 \\
(3.02)^{b}\end{array}$ & 25.47 & 30.62 \\
\hline C. blackburni & 11.34 & $\begin{array}{c}7.84 \\
(2.89)^{\mathrm{b}}\end{array}$ & 12.40 & $\begin{array}{l}8.15 \\
(2.94)^{\mathrm{b}}\end{array}$ & 30.86 & 34.27 \\
\hline NSKE $5 \%$ & 11.24 & $\begin{array}{c}8.13 \\
(2.94)^{\mathrm{c}}\end{array}$ & 11.80 & $\begin{array}{c}8.42 \\
(2.99)^{\mathrm{b}}\end{array}$ & 27.67 & 28.64 \\
\hline $\begin{array}{l}\text { T. chilonis }+ \\
\text { B. brevicornis }\end{array}$ & 10.12 & $\begin{array}{c}7.55 \\
(2.83)^{\mathrm{b}}\end{array}$ & 12.40 & $\begin{array}{c}7.88 \\
(2.90)^{\mathrm{a}}\end{array}$ & 25.40 & 36.45 \\
\hline $\begin{array}{l}\text { C. blackburni }+ \\
\text { B. brevicornis }\end{array}$ & 11.65 & $\begin{array}{c}7.30 \\
(2.79)^{\mathrm{ab}}\end{array}$ & 12.60 & $\begin{array}{c}7.54 \\
(2.84)^{\mathrm{a}}\end{array}$ & 37.34 & 40.16 \\
\hline $\begin{array}{l}\text { NSKE } 5 \%+ \\
\text { B. brevicornis }\end{array}$ & 10.58 & $\begin{array}{c}7.89 \\
(2.89)^{\mathrm{b}}\end{array}$ & 11.80 & $\begin{array}{c}7.89 \\
(2.90)^{\mathrm{a}}\end{array}$ & 25.43 & 33.14 \\
\hline $\begin{array}{l}\text { T. chilonis }+ \\
\text { C. blackburni }+ \\
\text { B. brevicornis }\end{array}$ & 11.12 & $\begin{array}{l}6.60 \\
(2.66)^{\mathrm{a}}\end{array}$ & 12.50 & $\begin{array}{c}7.33 \\
(2.80)^{\mathrm{a}}\end{array}$ & 40.65 & 41.36 \\
\hline $\begin{array}{l}\text { Endosulfan } \\
35 \mathrm{EC}\end{array}$ & 10.87 & $\begin{array}{c}6.56 \\
(2.66)^{\mathrm{a}}\end{array}$ & 11.9 & $\begin{array}{c}7.12 \\
(2.76)^{\mathrm{a}}\end{array}$ & 39.65 & 40.17 \\
\hline Untreated check & 10.24 & $\begin{array}{c}10.55 \\
(3.32)^{\mathrm{d}}\end{array}$ & 11.3 & $\begin{array}{l}11.55 \\
(3.47)^{\mathrm{c}}\end{array}$ & - & - \\
\hline
\end{tabular}

* Mean of three replications

** Pooled mean of three rounds of releases/spray; PTC $=$ pretreatment count 
In a column, means followed by same letter(s) are not significantly different by DMRT $(\mathrm{P}<0.05)$

In the second trial, observations on the population of E. vittella and $H$. armigera revealed similar trends as that of first trial (Table 4). Significantly lower population of E. vittella and $H$. armigera was recorded in the treatments involving the combination of $T$. chilonis $+C$. blackburni + B. brevicornis and C. blackburni + B. brevicornis which was comparable to that of endosulfan 35 EC. Sangwan (1972) reported the efficacy of Bracon kirkpatricki at 3000 adults per ha and $T$. brasiliensis at 1, 50,000 adults ha ${ }^{-1}$ under field condition against $H$. armigera and Earias spp. on cotton. Efficacy of C. blackburni, B. kirkpatricki and Trichogramma spp. against Earias spp. and H. armigera on cotton was also reported by earlier workers (Pawar and Prasad, 1988) in agreement with the present findings. Agarwal and Gupta (1986) and Forehand et al., (2006) reported that mass releases of egg parasitoid $T$. chilonis and T. acheae, egg larval parasitoid, C. blackburni and larval parasitoid $B$. kirkpatriki during square formation stage reduced the incidence of all three species of bollworms in cotton.

Table 4. Efficacy of braconid parasitoids against larval population of okra fruit borers: trial II (Kozhimadai, Coimbatore 2007 - 08)

\begin{tabular}{|c|c|c|c|c|c|c|}
\hline \multirow[t]{2}{*}{ Treatment* } & \multicolumn{4}{|c|}{$\begin{array}{l}\text { No. of larvae } / 10 \text { plants } \\
\text { (pooled mean) } * *\end{array}$} & \multicolumn{2}{|c|}{$\begin{array}{l}\text { \% reduction over } \\
\text { PTC (due to } \\
\text { parasitization / } \\
\text { chemicals) }\end{array}$} \\
\hline & PTC & $\begin{array}{c}E . \\
\text { vittella }\end{array}$ & PTC & $\begin{array}{c}H . \\
\text { armigera }\end{array}$ & $\begin{array}{c}E . \\
\text { vittella }\end{array}$ & $\begin{array}{c}\mathrm{H} . \\
\text { armigera }\end{array}$ \\
\hline B. brevicornis & 7.91 & $\begin{array}{c}5.30 \\
(2.41)^{\mathrm{c}}\end{array}$ & 11.51 & $\begin{array}{c}6.80 \\
(2.70)^{\mathrm{b}}\end{array}$ & 33.00 & 40.92 \\
\hline T. chilonis & 8.65 & $\begin{array}{c}6.33 \\
(2.61)^{\mathrm{d}}\end{array}$ & 11.48 & $\begin{array}{c}8.19 \\
(2.95)^{\mathrm{d}}\end{array}$ & 26.82 & 28.66 \\
\hline C. blackburni & 7.80 & $\begin{array}{c}5.15 \\
(2.38)^{\mathrm{c}}\end{array}$ & 11.44 & $\begin{array}{c}6.68 \\
(2.68)^{\mathrm{ab}}\end{array}$ & 33.97 & 41.61 \\
\hline NSKE 5\% & 8.18 & $\begin{array}{c}5.66 \\
(2.48)^{\mathrm{c}}\end{array}$ & 11.32 & $\begin{array}{c}7.33 \\
(2.80)^{\mathrm{c}}\end{array}$ & 30.81 & 35.25 \\
\hline $\begin{array}{l}\text { T. chilonis }+ \\
\text { B. brevicornis }\end{array}$ & 7.84 & $\begin{array}{c}4.82 \\
(2.31)^{\mathrm{b}}\end{array}$ & 11.12 & $\begin{array}{c}6.41 \\
(2.63)^{\mathrm{ab}}\end{array}$ & 38.52 & 42.36 \\
\hline $\begin{array}{l}\text { C. blackburni+ } \\
\text { B. brevicornis }\end{array}$ & 8.32 & $\begin{array}{c}4.42 \\
(2.22)^{\mathrm{b}}\end{array}$ & 12.21 & $\begin{array}{c}6.14 \\
(2.58)^{\mathrm{ab}}\end{array}$ & 46.88 & 49.71 \\
\hline $\begin{array}{l}\text { NSKE } 5 \%+ \\
\text { B. brevicornis }\end{array}$ & 7.97 & $\begin{array}{c}4.59 \\
(2.26)^{\mathrm{b}}\end{array}$ & 11.27 & $\begin{array}{c}6.31 \\
(2.61)^{\mathrm{ab}}\end{array}$ & 42.41 & 44.01 \\
\hline $\begin{array}{l}\text { T. chilonis }+ \\
\text { C. blackburni }+ \\
\text { B. brevicornis }\end{array}$ & 8.24 & $\begin{array}{c}4.26 \\
(2.18)^{\mathrm{b}}\end{array}$ & 12.01 & $\begin{array}{c}5.94 \\
(2.54)^{\mathrm{a}}\end{array}$ & 48.30 & 50.54 \\
\hline $\begin{array}{l}\text { Endosulfan } \\
35 \mathrm{EC}\end{array}$ & 8.04 & $\begin{array}{c}3.73 \\
(2.06)^{\mathrm{a}}\end{array}$ & 11.81 & $\begin{array}{c}5.58 \\
(2.47)^{\mathrm{a}}\end{array}$ & 53.61 & 52.75 \\
\hline Untreated check & 8.25 & $\begin{array}{c}8.59 \\
(3.02)^{\mathrm{e}}\end{array}$ & 12.35 & $\begin{array}{l}12.96 \\
(3.67)^{\mathrm{e}}\end{array}$ & - & - \\
\hline
\end{tabular}




\section{Fruit borer damage and yield}

The per cent fruit damage recorded on both number and weight basis revealed significant differences among the treatments compared to the untreated check in both the trials (Tables 5 and 6). In the first trial, combination of T. chilonis $+C$. blackburni $+B$. brevicornis and $C$. blackburni + B. brevicornis recorded significantly lower per cent damage on number basis and weight basis after three releases/spray. Significantly higher per cent reduction over control on both number (42.70 and $40.69 \%$ ) and weight basis (47.32 and $45.00 \%)$ were recorded in the same treatments. This was next in the order of efficacy to endosulfan $35 \mathrm{EC}$ which recorded 45.06 and 54.49 per cent reduction over control on both number and weight basis, respectively (Table 5).

Table 5. Efficacy of braconid parasitoids against fruit borer damage on number and weight basis: trial I (Vellimalaipatinam, Coimbatore, 2007- 08)

\begin{tabular}{|c|c|c|c|c|}
\hline \multirow{2}{*}{ Treatment } & \multicolumn{2}{|c|}{$\begin{array}{l}\% \text { fruit damage } * * \\
\text { (pooled mean)*** }\end{array}$} & \multicolumn{2}{|c|}{$\begin{array}{l}\text { \% reduction } \\
\text { over control }\end{array}$} \\
\hline & $\begin{array}{c}\text { Number } \\
\text { basis }\end{array}$ & $\begin{array}{c}\text { Weight } \\
\text { basis }\end{array}$ & $\begin{array}{c}\text { Number } \\
\text { basis }\end{array}$ & $\begin{array}{c}\text { Weight } \\
\text { basis }\end{array}$ \\
\hline B. brevicornis & $\begin{array}{c}7.79 \\
(16.20)^{\mathrm{d}}\end{array}$ & $\begin{array}{c}6.63 \\
(14.92)^{\mathrm{d}}\end{array}$ & 31.96 & 33.09 \\
\hline T. chilonis & $\begin{array}{c}8.28 \\
(16.72)^{\mathrm{e}}\end{array}$ & $\begin{array}{c}7.46 \\
(15.86)^{\mathrm{e}}\end{array}$ & 27.69 & 24.72 \\
\hline C. blackburni & $\begin{array}{c}7.56 \\
(15.96)^{\mathrm{cd}}\end{array}$ & $\begin{array}{c}6.37 \\
(14.62)^{\mathrm{c}}\end{array}$ & 33.98 & 35.72 \\
\hline NSKE $5 \%$ & $\begin{array}{c}7.98 \\
(16.40)^{\mathrm{d}}\end{array}$ & $\begin{array}{c}6.92 \\
(15.25)^{\mathrm{e}}\end{array}$ & 30.30 & 30.17 \\
\hline $\begin{array}{l}\text { T. chilonis }+ \\
\text { B. brevicornis }\end{array}$ & $\begin{array}{c}7.27 \\
(15.64)^{\mathrm{cd}}\end{array}$ & $\begin{array}{c}6.30 \\
(14.53)^{\mathrm{c}}\end{array}$ & 36.50 & 36.42 \\
\hline $\begin{array}{l}\text { C. blackburni }+ \\
\text { B. brevicornis }\end{array}$ & $\begin{array}{c}6.79 \\
(15.10)^{\mathrm{a}}\end{array}$ & $\begin{array}{c}5.45 \\
(13.50)^{\mathrm{b}}\end{array}$ & 40.69 & 45.00 \\
\hline $\begin{array}{l}\text { NSKE } 5 \%+ \\
\text { B. brevicornis }\end{array}$ & $\begin{array}{c}7.05 \\
(15.40)^{b}\end{array}$ & $\begin{array}{c}5.99 \\
(14.17)^{\mathrm{c}}\end{array}$ & 38.43 & 39.55 \\
\hline $\begin{array}{l}\text { T. chilonis }+ \\
\text { C. blackburni }+ \\
\text { B. brevicornis }\end{array}$ & $\begin{array}{c}6.56 \\
(14.84)^{\mathrm{a}}\end{array}$ & $\begin{array}{c}5.22 \\
(13.20)^{b}\end{array}$ & 42.70 & 47.32 \\
\hline $\begin{array}{l}\text { Endosulfan } \\
35 \mathrm{EC}\end{array}$ & $\begin{array}{c}6.29 \\
(14.53)^{\mathrm{a}}\end{array}$ & $\begin{array}{c}4.51 \\
(12.26)^{\mathrm{a}}\end{array}$ & 45.06 & 54.49 \\
\hline Untreated check & $\begin{array}{c}11.45 \\
(19.78)^{\mathrm{f}}\end{array}$ & $\begin{array}{c}9.91 \\
(18.35)^{\mathrm{f}}\end{array}$ & - & - \\
\hline
\end{tabular}

In the second field trial, the per cent fruit borer damage, also followed similar trends as that of larval population (Table 6). Significantly higher per cent reduction in the fruit borer damage both number (46.01 and $44.85 \%)$ and weight basis (54.78 and $47.52 \%)$ were observed with the treatment involving combination of T. chilonis $+C$. blackburni $+B$. brevicornis and C. blackburni + B. brevicornis which was next to Endosulfan $35 \mathrm{EC}$ in the order of efficacy. Dhane et al., (2007) stated that innundative release of C. blackburni at 75,000 adults $\mathrm{ha}^{-1}$ resulted in minimum fruit borer infestation in both number $(11.5 \%)$ and 
weight basis (11.31\%). Among the treatments, release of two or three parasitoid species in combination was found to be more effective against fruit borer than releasing individual parasitoid species.

Table 6. Efficacy of braconid parasitoids against okra fruit borer damage on number and weight basis: trial II (Kozhimadai, Coimbatore, 2007- 08)

\begin{tabular}{|c|c|c|c|c|}
\hline \multirow{2}{*}{ Treatment } & \multicolumn{2}{|c|}{$\begin{array}{l}\% \text { fruit damage } \\
\text { (pooled mean)*** }\end{array}$} & \multicolumn{2}{|c|}{$\begin{array}{l}\text { \% reduction } \\
\text { over control }\end{array}$} \\
\hline & $\begin{array}{c}\text { Number } \\
\text { basis }\end{array}$ & $\begin{array}{c}\text { Weight } \\
\text { basis }\end{array}$ & $\begin{array}{c}\text { Number } \\
\text { basis }\end{array}$ & $\begin{array}{l}\text { Weight } \\
\text { basis }\end{array}$ \\
\hline B. brevicornis & $\begin{array}{c}6.58 \\
(14.76)^{g}\end{array}$ & $\begin{array}{c}7.07 \\
(15.39)^{\mathrm{g}}\end{array}$ & 38.83 & 39.74 \\
\hline T. chilonis & $\begin{array}{c}6.76 \\
(14.97)^{\mathrm{i}}\end{array}$ & $\begin{array}{c}7.54 \\
(15.92)^{\mathrm{i}}\end{array}$ & 37.16 & 35.69 \\
\hline C. blackburni & $\begin{array}{c}6.46 \\
(14.63)^{\mathrm{f}}\end{array}$ & $\begin{array}{c}6.86 \\
(15.16)^{\mathrm{f}}\end{array}$ & 39.96 & 41.51 \\
\hline NSKE 5\% & $\begin{array}{c}6.65 \\
(14.83)^{\mathrm{h}}\end{array}$ & $\begin{array}{c}7.28 \\
(15.63)^{\mathrm{h}}\end{array}$ & 38.22 & 37.94 \\
\hline $\begin{array}{l}\text { T. chilonis }+ \\
\text { B. brevicornis }\end{array}$ & $\begin{array}{c}6.24 \\
(14.38)^{\mathrm{e}}\end{array}$ & $\begin{array}{c}6.66 \\
(14.92)^{\mathrm{e}}\end{array}$ & 42.04 & 43.28 \\
\hline $\begin{array}{l}\text { C. blackburni }+ \\
\text { B. brevicornis }\end{array}$ & $\begin{array}{c}5.94 \\
(14.01)^{\mathrm{c}}\end{array}$ & $\begin{array}{c}6.16 \\
(14.32)^{\mathrm{c}}\end{array}$ & 44.85 & 47.52 \\
\hline $\begin{array}{l}\text { NSKE } 5 \%+ \\
\text { B. brevicornis }\end{array}$ & $\begin{array}{c}6.12 \\
(14.23)^{\mathrm{d}}\end{array}$ & $\begin{array}{c}6.45 \\
(14.68)^{\mathrm{d}}\end{array}$ & 43.18 & 45.02 \\
\hline $\begin{array}{l}\text { T. chilonis + } \\
\text { C. blackburni } \\
+ \text { B. brevicornis }\end{array}$ & $\begin{array}{c}5.81 \\
(13.85)^{\mathrm{b}}\end{array}$ & $\begin{array}{c}5.30 \\
(13.25)^{\mathrm{b}}\end{array}$ & 46.01 & 54.78 \\
\hline Endosulfan $35 \mathrm{EC}$ & $\begin{array}{c}5.52 \\
(13.49)^{\mathrm{a}}\end{array}$ & $\begin{array}{c}4.89 \\
(12.65)^{\mathrm{a}}\end{array}$ & 48.71 & 58.33 \\
\hline Untreated check & $\begin{array}{c}10.76 \\
(19.15)^{\mathrm{j}} \\
\end{array}$ & $\begin{array}{c}11.74 \\
(20.03)^{j}\end{array}$ & - & - \\
\hline
\end{tabular}

In the field trial conducted at Vellimalaipatinam village, the highest yields of 5480 and 5450 $\mathrm{kg} \mathrm{ha}^{-1}$ were recorded in the treatments $T$. chilonis $+C$. blackburni + B. brevicornis and $C$. blackburni + B. brevicornis which were on par with endosulfan 35 EC $\left(5467 \mathrm{~kg} \mathrm{ha}^{-1}\right)$. Combination of $C$. blackburni $+B$. brevicornis recorded significantly higher cost benefit ratio of 1:2.18 followed by 1:2.17 in T. chilonis + C. blackburni + B. brevicornis (Table 7).

In the second field trial, the treatment, T. chilonis + C. blackburni + B. brevicornis recorded significantly higher yield of $6250 \mathrm{~kg} \mathrm{ha}^{-1}$ followed by C. blackburni + B. brevicornis $(6200$ $\left.\mathrm{kg} \mathrm{ha}^{-1}\right)$ which were next to Endosulfan $35 \mathrm{EC}\left(6565 \mathrm{~kg} \mathrm{ha}^{-1}\right)$ in the order of efficacy. Among the parasitoid combination, C. blackburni + B. brevicornis recorded the cost benefit ratio of 1:2.48 followed by $T$. chilonis + C. blackburni + B. brevicornis (1:2.47). However, the highest cost benefit ratio of 1:2.80 was recorded in Endosulfan $35 \mathrm{EC}$ treatment(Table 8). 
Table 7. Efficacy of braconid parasitoids on okra yield and cost benefit ratio: Trial I Vellimalaipatinam, Coimbatore, 2007-2008

\begin{tabular}{|c|c|c|c|c|c|}
\hline Treatment & $\begin{array}{c}\text { Yield of } \\
\text { healthy } \\
\text { fruits } \\
\text { (kg/ha)* }\end{array}$ & $\begin{array}{c}\text { Gross } \\
\text { income } \\
\text { (Rs./ha) }\end{array}$ & $\begin{array}{c}\text { Cost of } \\
\text { protection } \\
\text { (Rs./ha) }\end{array}$ & $\begin{array}{c}\text { Cost of } \\
\text { production } \\
(\text { Rs./ha') }\end{array}$ & $\begin{array}{l}\mathrm{C}: \mathrm{B} \\
\text { ratio }\end{array}$ \\
\hline B. brevicornis & $4786^{\mathrm{f}}$ & 38288 & 2200 & 19950 & $1: 1.92$ \\
\hline T. chilonis & $4626^{\mathrm{de}}$ & 37005 & 550 & 18300 & $1: 2.02$ \\
\hline C. blackburni & $5115^{\mathrm{d}}$ & 40920 & 2200 & 19950 & $1: 2.05$ \\
\hline NSKE $5 \%$ & $4683^{e}$ & 37464 & 1600 & 19350 & $1: 1.94$ \\
\hline $\begin{array}{l}\text { T. chilonis }+ \\
\text { B. brevicornis }\end{array}$ & 5148 & 41180 & 1375 & 19125 & $1: 2.15$ \\
\hline $\begin{array}{l}\text { C. blackburni }+ \\
\text { B. brevicornis }\end{array}$ & $5450^{\mathrm{ab}}$ & 43600 & 2200 & 19950 & $1: 2.18$ \\
\hline $\begin{array}{l}\text { NSKE } 5 \%+ \\
\text { B. brevicornis }\end{array}$ & $5242^{\mathrm{ab}}$ & 41936 & 1800 & 19550 & $1: 2.15$ \\
\hline $\begin{array}{l}\text { T. chilonis }+ \\
\text { C. blackburni }+ \\
\text { B. brevicornis }\end{array}$ & $5480^{\mathrm{a}}$ & 43840 & 2425 & 20175 & $1: 2.17$ \\
\hline $\begin{array}{l}\text { Endosulfan } \\
35 \mathrm{EC}\end{array}$ & $5467^{\mathrm{a}}$ & 43736 & 1000 & 18750 & $1: 2.33$ \\
\hline Untreated check & $3216^{\mathrm{g}}$ & 25278 & - & 17750 & - \\
\hline
\end{tabular}

* Mean of three replications

Cost of fruits $=$ Rs. 8 per kg (note: Rs. $=$ Indian Rs.)

Cost of production $=$ cost of protection + cost of cultivation @ Rs. 17750 ha $^{-1}$

In a column, means followed by same letter(s) are not significantly different by DMRT $(\mathrm{P}=0.05)$

Sangwan (1972) and Dhandapani et al., (1992) reported that the utilization of biocontrol agents increased the seed cotton yield with highest cost benefit ratio. It is in confirmation with the present findings. However, increased yield coupled with highest cost benefit ratio in insecticide treated plots may not have long term benefits while considering environmental safety. 
Table 8. Efficacy of braconid parasitoids on okra yield and cost benefit ratio: trial II (Kozhimadai, Coimbatore, 2007-08)

\begin{tabular}{|c|c|c|c|c|c|}
\hline Treatment & $\begin{array}{c}\text { Yield of } \\
\text { healthy fruits } \\
\left(\mathrm{kg} \mathrm{ha}^{-1}\right)^{*}\end{array}$ & $\begin{array}{c}\text { Gross } \\
\text { income } \\
\left(\mathrm{Rs} \mathrm{ha}^{-1}\right) \\
\end{array}$ & $\begin{array}{c}\text { Cost of } \\
\text { Protecttion } \\
\left(\mathrm{Rs} \mathrm{ha}^{-1}\right) \\
\end{array}$ & $\begin{array}{c}\text { Cost of } \\
\text { Production } \\
\left(\mathrm{Rs} \mathrm{ha}^{-1}\right) \\
\end{array}$ & $\begin{array}{c}\mathrm{C}: \mathrm{B} \\
\text { ratio }\end{array}$ \\
\hline B. brevicornis & $4920^{f}$ & 39360 & 2200 & 19950 & $1: 1.97$ \\
\hline T. chilonis & $4524^{\mathrm{g}}$ & 36192 & 550 & 18300 & $1: 1.98$ \\
\hline C. blackburni & $5150^{\mathrm{e}}$ & 41200 & 2200 & 19950 & $1: 2.07$ \\
\hline NSKE $5 \%$ & $4650^{\mathrm{fg}}$ & 37200 & 1600 & 19350 & $1: 1.92$ \\
\hline $\begin{array}{l}\text { T. chilonis }+ \\
\text { B. brevicornis }\end{array}$ & $5750^{d}$ & 46000 & 1375 & 19125 & $1: 2.40$ \\
\hline $\begin{array}{l}\text { C. blackburni }+ \\
\text { B. brevicornis }\end{array}$ & $6200^{b}$ & 49600 & 2200 & 19950 & $1: 2.48$ \\
\hline $\begin{array}{l}\text { NSKE } 5 \%+ \\
\text { B. brevicornis }\end{array}$ & $5955^{\mathrm{c}}$ & 47640 & 1800 & 19550 & $1: 2.44$ \\
\hline $\begin{array}{l}\text { T. chilonis }+ \\
\text { C. blackburni }+ \\
\text { B. brevicornis }\end{array}$ & $6250^{\mathrm{b}}$ & 50000 & 2425 & 20175 & $1: 2.47$ \\
\hline $\begin{array}{l}\text { Endosulfan } \\
35 \text { EC }\end{array}$ & $6565^{\mathrm{a}}$ & 52520 & 1000 & 18750 & $1: 2.80$ \\
\hline Untreated check & $2850^{\mathrm{h}}$ & 22800 & - & 17750 & - \\
\hline
\end{tabular}

* Mean of three replications

Cost of fruits = Rs. 8 per kg (Note: Rs.= Indian Rs)

Cost of production $=$ cost of protection + cost of cultivation @ Rs. 17750 ha $^{-1}$

In a column, means followed by same letter(s) are not significantly different by DMRT $(\mathrm{P}=0.05)$

\section{CONCLUSIONS}

Under field conditions, pest stages will occur in a staggered manner due to overlapping generations. Hence, release of single parasitoid may not have the expected result as they target only particular stage of the pest. Hence, combination of two or three parasitoid species targeting different stages of the pest will be more viable. In the present finding, combination of $T$. chilonis $+C$. blackburni $+B$. brevicornis recorded significantly lower larval population and higher per cent reduction in fruit damage with increased yield than the combination of C. blackburni + B. brevicornis . However, the cost benefit ratio was high in combination of two parasitoid species than the three parasitoid species which may be due to the additional cost involved in the protection. Combined release of C. blackburni and B. brevicornis at fortnight intervals, starting from flower initiation may give long term benefits in the biosuppression of the fruit borers in okra in an ecofriendly manner. 


\section{ACKNOWLEDGEMENT}

The authors are grateful to TNAU, Coimbatore, CPCRI, Kayankulam, Kerala and the farmers of Vellimalaipatinam and Kozhimadai villages of Coimbatore district, Tamil Nadu for providing facilities for the successful conduct of the trials.

\section{REFERENCES}

Agarwal, R.A. and Gupta, G.P. (1986). Recent advances in cotton pest management. Plant Prot. Bull. 88(1-4): 375-378.

Akila, S. and Babu, P.C.S. (1994). Release of different dose of Trichogramma and its effect on internode borer, yield and quality of sugarcane. Sugarcane (London) 2: 22-23.

Atwal, A.S. and Singh, B. (1990). Pest population and assessment of crop losses, Publication. Indian Agriculture Research Institute, New Delhi.

Dhandapani, N., Kalyanasundaram, M., Swamiappan, M., Babu, P.C.S. and Jayaraj, S. (1992). Experiments on management of major pests of cotton with biocontrol agents in India. J. Appl. Entomol. 114: 52- 56.

Dhane, A.S., Ghodaki, B.S. and Babar, M.S. (2007). Field evaluation of Chelonus blackburni against shoot and fruit borer Earias vittella infesting okra. Indian J. Entomol. 69(1): 54-57.

FAO (2007). Agricultural production data base. Food and Agricultural Organization. http//:apps.fao.org./faostat./ 340p.

Forehand, L.M., Orr, D.B. and Linker, H.M. (2006). Evaluation of a commercially available beneficial insect for management of lepidopteron pests. J. Econ. Entomol. 99(3): 641-647.

Gopalakrishnan, T.R. (2007). Vegetable Crops, New India Publishing Agency, New Delhi.

Hopper, K.R. (1986). Preference, acceptance and fitness components of Microplitis croceipes (Hymenoptera: Braconidae) attacking various instars of Heliothis virescence (Lepidoptera: Noctuidae). Environ. Entomol. 15: 274-280.

Jagtab, C.R., Shetgar, S.S. and Nalwandikar, P.K. (2007). Fluctuation in population of lepidopterous pest infesting okra in relation to weather parameters during Kharif. Indian J. Entomol. 69(3): 218-220.

Jeyarani, S., Karuppuchamy, P. and Sathiah, N. (2008). Interaction between the egg larval parasitoid, Chelonus blackburni and Nucleopolyhedrovirus in Helicoverpa armigera (Hübner). pp.126-130. In: Ignachimuthu and Jayaraj, S. (Ed.). Recent trends in pest management. Elite publishing (Pvt.) Ltd. New Delhi.

Jhansi, K. (1984). Studies on Bracon hebetor (Say), a larval parasitoids of Maruca testulalis (Geyer). 96p. M. Sc. Thesis. Tamil Nadu Agric. Univ., Coimbatore. 
King, E.G., Powell, J.E. and Coleman, R.J. (1985). A high incidence of parasitism of Heliothis spp (Lepidoptera: Noctuidae) larvae in cotton in south-eastern Arkansas. Entomophaga 30: 419-426.

Mani, M., Krishnamoorthy, A. and Gopalakrishnan, C. (2005). Biological control of lepidopterous pests of Horticultural crops in India. A. Review. Agric. Res. 26(1): 39-49.

Pawar. A.D. and Prasad, J. (1988). Evaluation of some parasites in biocontrol of cotton bollworms in Haryana. Indian J. Plant Prot. 13(1): 21-24.

Priya, B.S. and Misra, H.P. (2007). Biopesticides for the management of okra fruit borer, Earias vittella (Fabricius). Pest Mgt. Horti. Ecosystem 13(2): 176-179.

Reichmuth, C.H., Scholler, M. and Ulrichs, C.H. (1997). Stored Product Research in Cereals. Verlag Thomas Mann, Gelsenkirchen, 119 p.

Sangwan, H.S. (1972). Some observations on Stenobracon deesae Cameron. Indian J. Entomol. 34: 172-173.

Sheeba, M. and Narendran, T.C. (2007). On the identity of two confusing species of (Braconidae: Hymenoptera) parasitic on Opisina arenosella (Walker). J. Plant. Crops 35(3): 195-197.

Snedecor, G.W. and Cochran, W.G. (1967). Statistical methods. Iowa state Univ. Press. Ames, Iowa, 462p.

Swamiappan, M. and Balasubramanian, M. (1979). Studies on mass multiplication and potentiality of Chelonus blackburni Cam a braconid parasitoid of cotton boll worms. Entomo. 5: 73-75. 